\title{
Theory of Asymmetric Electrostatic Force
}

\author{
Katsuo Sakai \\ Electrostatic Generator Research Laboratory, Yokohama, Japan \\ Email: gy7a-ski@asahi-net.or.jp
}

How to cite this paper: Sakai, K. (2017) Theory of Asymmetric Electrostatic Force. Journal of Electromagnetic Analysis and Applications, 9, 34-42.

https://doi.org/10.4236/jemaa.2017.92004

Received: January 6, 2017

Accepted: February 12, 2017

Published: February 15, 2017

Copyright $\odot 2017$ by author and Scientific Research Publishing Inc. This work is licensed under the Creative Commons Attribution International License (CC BY 4.0).

http://creativecommons.org/licenses/by/4.0/

\begin{abstract}
Asymmetric Electrostatic Force is a very interesting phenomenon. The intensity of the electrostatic force that acts on a charged asymmetric shaped conductor changes when the direction of the electric field is reversed. The theory of this phenomenon is not clear until today. Therefore this paper will clearly explain the theory of this phenomenon with Gauss's law. Total $\Sigma E S$ on a closed Gaussian surface around a disk-cylinder shaped charged conductor is the same before and after when the direction of the electric field is reversed. However the distribution of $\Sigma E S$ changes. Many part of total $\Sigma E S$ gather on the front surface of the disk when the electric field is generated. But they transfer to the peripheral side of the cylinder when the direction of the electric field is reversed. This result means that many charges transferred from the surface to the peripheral side. As a result the intensity of the field direction electrostatic force becomes weak.
\end{abstract}

\section{Keywords}

Electrostatic Force, Asymmetric Electrostatic Force,

Asymmetric Shaped Conductor, Charge Distribution, Gauss's Law

\section{Introduction}

Historically, the main application of electrostatics has been Xerography and powder coating. The main materials used in these technologies are charged powders. The electrostatic force that acts on this powder has been calculated using the following Coulomb equation:

$$
F=q E
$$

where $F$ is Electrostatic force, $q$ is Charge quantity, $E$ is Intensity of electric field.

This equation can be applied to a point charge and a sphere-shaped charge carrier [1], and a charged powder can be treated as a point charge. It is apparent from this equation that the intensity of the electrostatic force that acts on the charged powder does not change when the direction of the electric field is re- 
versed.

On the contrary the intensity of the electrostatic force that acts on an asymmetric shaped conductor changed when the direction of the electric field was reversed. This interesting new phenomenon was reported depending on simple simulation and experiment. And it was unofficially named as Asymmetric Electrostatic Force [2]. After that, several simulations and experiments have been performed [3] [4] [5] [6]. And finally the existence of the Asymmetric Electrostatic Force was almost confirmed [7].

However the explain of the theory of Asymmetric Electrostatic Force has been not clear until today.

Therefore the purpose of this paper is to explain the theory clearly by Gauss's law.

\section{Simulation}

\subsection{Simulation Method}

Figure 1 shows a schematic layout for the simulation. The circular electrodes and the disk-cylinder conductor ere lined up on the $\mathrm{Z}$ axis.

The radius of the electrodes was $54.1 \mathrm{~mm}$ and the distance between them was $106.9 \mathrm{~mm}$. The radius of the disk was $5.1 \mathrm{~mm}$ and its thickness was $0.2 \mathrm{~mm}$. The length of the cylinder was $5.1 \mathrm{~mm}$ and the outer and inner radii of the cylinder ware $2.8 \mathrm{~mm}$ and $2.6 \mathrm{~mm}$ respectively.

When positive high voltage is applied to the right electrode and the left electrode is grounded, an electric field is generated between the electrodes. This electric field is called as the forward electric field from here. On the contrary, when positive high voltage is applied to the left electrode and the right electrode is grounded, another electric field is generated between the electrodes. This

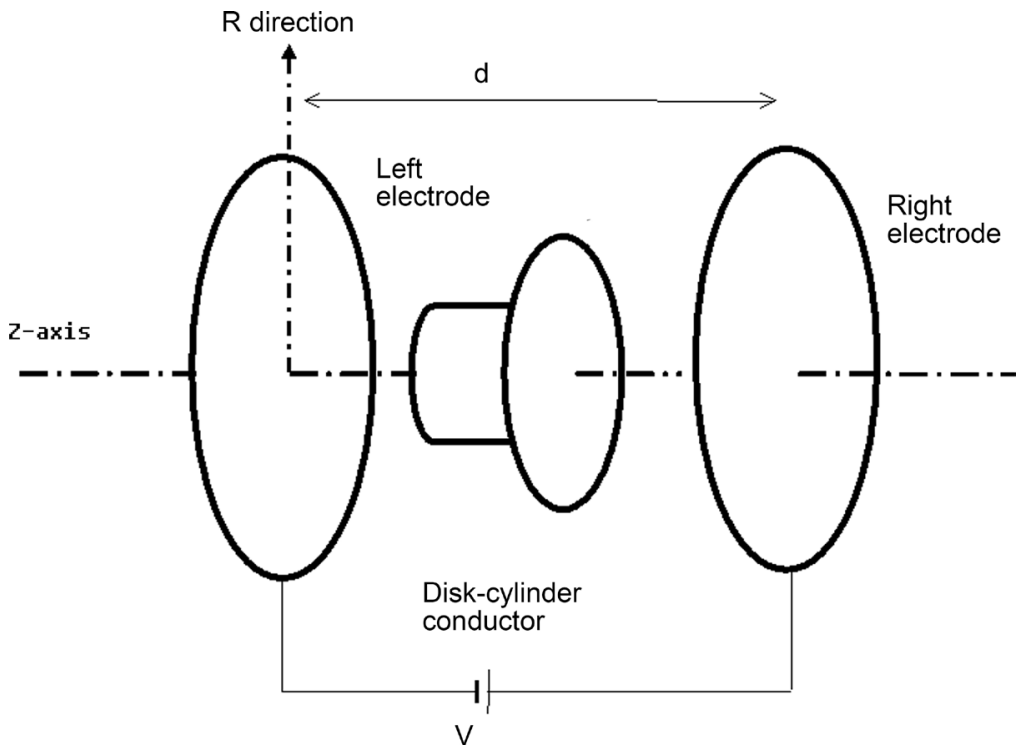

Figure 1. Schematic layout for simulating the electrostatic force acting on a charged disk-cylinder conductor in two different electric field. The directions of them are reversed each other. 
electric field is called as the backward electric field from here.

The charged disk-cylinder conductor is electrically floated.

Simulation target space was divided into small 1750 cells. Figure 2 shows some cells that ware placed around the disk-cylinder conductor. And Figure 3 shows an expounded picture of the cells that were placed at the center of the disk.

In Figure 2 and Figure 3, the red bold lines shows the surface of the disk-cylinder

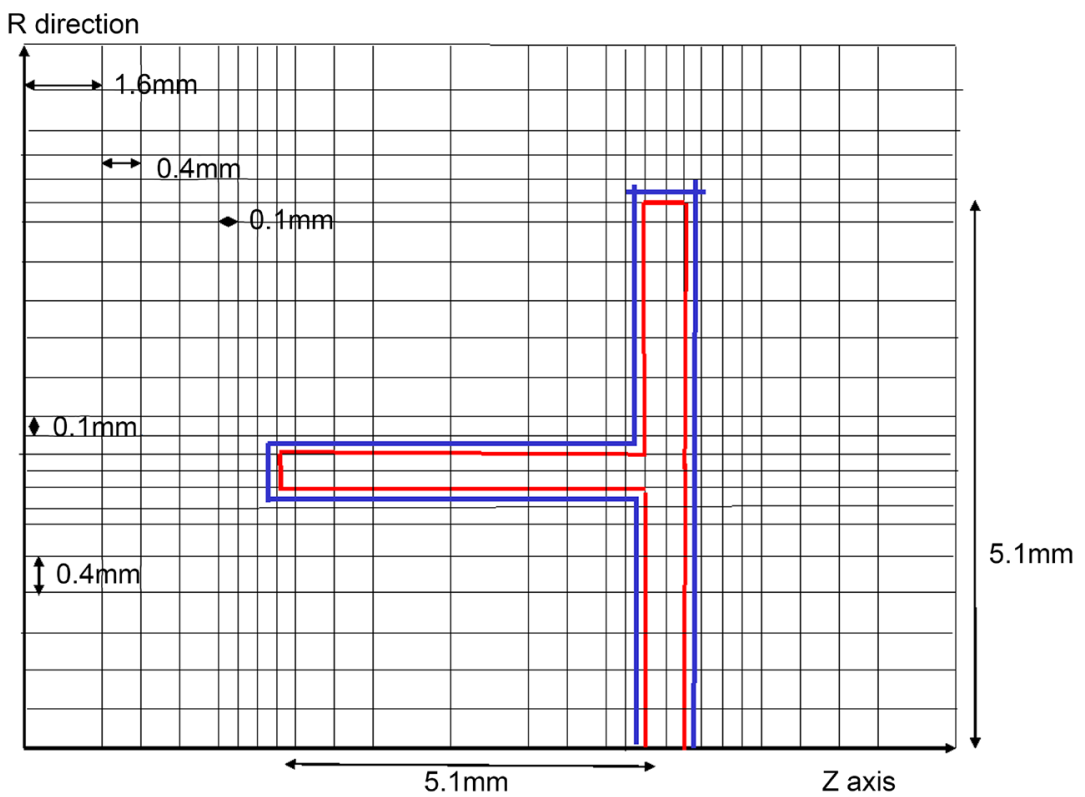

Figure 2. Cell layout around the disk-cylinder conductor for the axis symmetric finite element method.

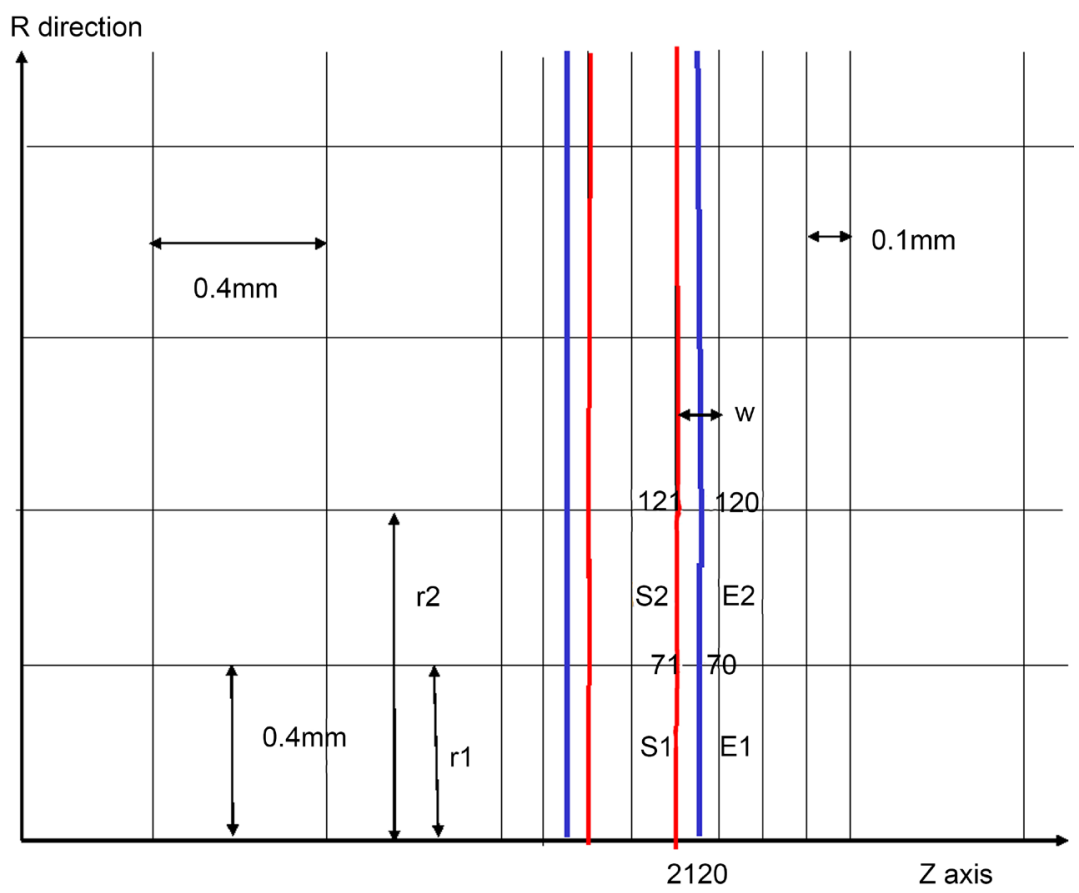

Figure 3. An expounded picture of the cells that were placed at the center of the disk. 
conductor. And the blue bold line shows a closed Gaussian surface that surrounds the disk-cylinder conductor. The distance between the surface of the disk-cylinder conductor and the Gaussian surface is $50 \mu \mathrm{m}$.

The electric potentials of all 1750 cell points were calculated by Axis symmetric Finite Element Method (FEM). This method can simulate the three-dimensional world. This is mathematically a two-dimensional program, but this is physically a three-dimensional program. Of course, this program is limited for simulating an axis-symmetric material. This method is well known in science and technology fields. Therefore the detail of the calculation is not explained in this paper.

FEM was introduced to Japan by Dr. Matsubara [8] [9] [10]. Then a FEM program for simulation of this research was made from his papers. The reliability of this program was confirmed by simulating a well-known subject that can be solved analytically [7].

The closed Gaussian surface shown by bold blue lines in Figure 2 consist of 56 different areas. They have different area $S_{\mathrm{k}}$ and different electric field $E_{\mathrm{k}}$ each other. However total of $E_{\mathrm{k}}{ }^{*} S_{\mathrm{k}}$ becomes constant always. And it can be calculated by the following equation (Gauss's law) [11].

$$
\sum_{k=1}^{56} E_{k} S_{k}=q / \varepsilon_{0}
$$

where $E_{\mathrm{k}}$ is the intensity of the electric field that through a small area of the Gaussian surface, $S_{\mathrm{k}}$ is area of a small area of the Gaussian surface, $q$ is quantity of charge that is given to the disk-cylinder conductor, $\varepsilon_{0}$ is Permittivity constant.

For example area $S_{1}$ and $S_{2}$ shown in Figure 3 are calculated by the following equations.

$$
\begin{gathered}
S_{1}=\pi \times r_{1}^{2} \\
S_{2}=\pi \times r_{2}^{2}-\pi \times r_{1}^{2}
\end{gathered}
$$

where $r_{\mathrm{k}}$ is outer radius of area $k$.

The intensity of the electric field $E_{1}$ and $E_{2}$ are calculated by the following equations.

$$
\begin{aligned}
& E_{1}=\frac{\frac{\left(V_{20}+V_{70}\right)}{2}-\frac{\left(V_{21}+V_{71}\right)}{2}}{w} \\
& E_{2}=\frac{\left(\frac{\left(V_{70}+V_{120}\right)}{2}-\frac{\left(V_{71}+V_{121}\right)}{2}\right)}{w}
\end{aligned}
$$

where $V_{\mathrm{k}}$ is simulated voltages of each cell points, $w$ is width of the cell.

The front surface of the disk has 18 different areas as shown in Figure 2. Therefore $\sum E S_{d f s}$ of the front surface of the disk is calculated by the following equation.

$$
\sum E S_{d f s}=\sum_{k=1}^{18} E_{k} S_{k}
$$


where $E_{\mathrm{k}}$ is the intensity of the electric field that through a small area of the Gaussian surface, $S_{\mathrm{k}}$ is area of a small area of the Gaussian surface, $k$ is number of the small area of the disk-cylinder conductor.

And $\Sigma E S$ of other parts can be calculated with the same method.

\subsection{Simulation Result of the Disk Conductor and the Cylinder Conductor}

Before simulating $\Sigma E S$ of the disk-cylinder conductor, $\Sigma E S$ of the lonely disk and $\Sigma E S$ of the lonely cylinder were simulated separately. $-0.8854 \mathrm{nC}$ was selected as the Charge quantity of them. Because the value of total $\Sigma E S\left(=q / \varepsilon_{0}\right)$ became just 100 always. And +40622 volts was selected as the high voltage that was applied to right or left electrode. The intensity of the electric field became $380000 \mathrm{~V} / \mathrm{m}$.

Figure 4 shows the simulation results of the lonely disk conductor. Total $\Sigma$ ES did not change, they were just 100 in the forward electric field and the backward electric field. However, $76 \%$ of them was occupied by the front surface in the forward electric field. On the contrary, $76 \%$ of them was occupied by the back surface in the backward electric field. And $12 \%$ of them was occupied by the back surface in the forward electric field and $12 \%$ of them was occupied by the front surface in the backward electric field. And $12 \%$ of them remained on the circumference side surface in the both electric field. As a result, the distribution pattern of $\Sigma E S$ was right and left symmetric.

Figure 5 shows the simulation result of the lonely cylinder. Total $\Sigma \mathrm{ES}$ was just 100 in the both electric field. And only $11 \%$ of them was occupied by the circular front edge in the forward electric field and only $11 \%$ was occupied by the circular back edge in the backward electric field. And $\Sigma E S$ of the circular back edge in the forward electric field and $\Sigma \mathrm{ES}$ of the front circular edge in the backward electric field were $0 \%$. As a result, $89 \%$ of them remained in the circular side surface in the both electric field. Then the distribution pattern of $\Sigma E S$ was right and left symmetric.

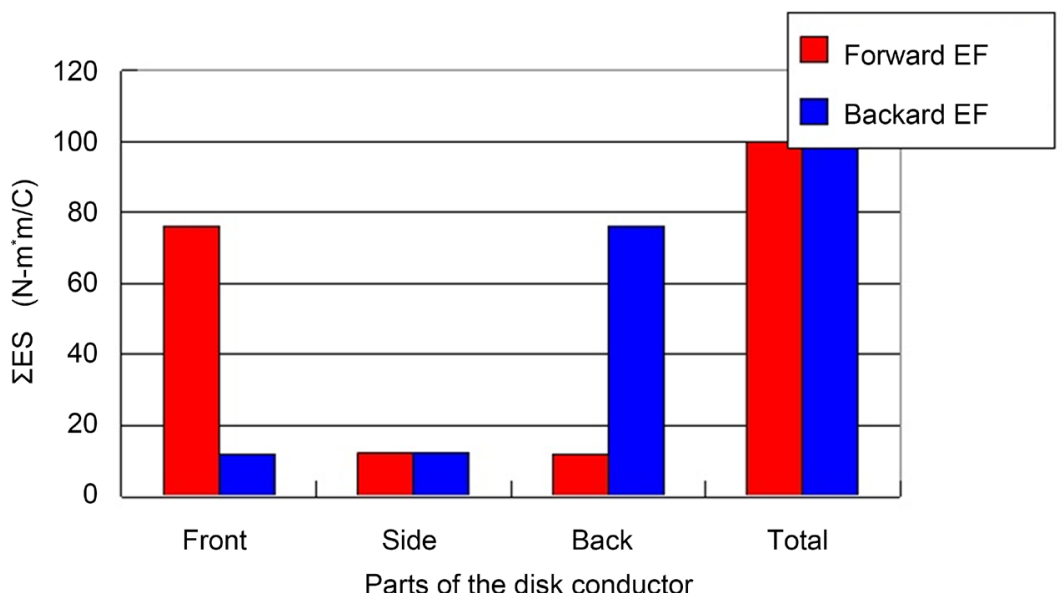

Figure 4. $\Sigma E S$ of each parts of the lonely disk shaped charged conductor in the forward and backward electric field. 


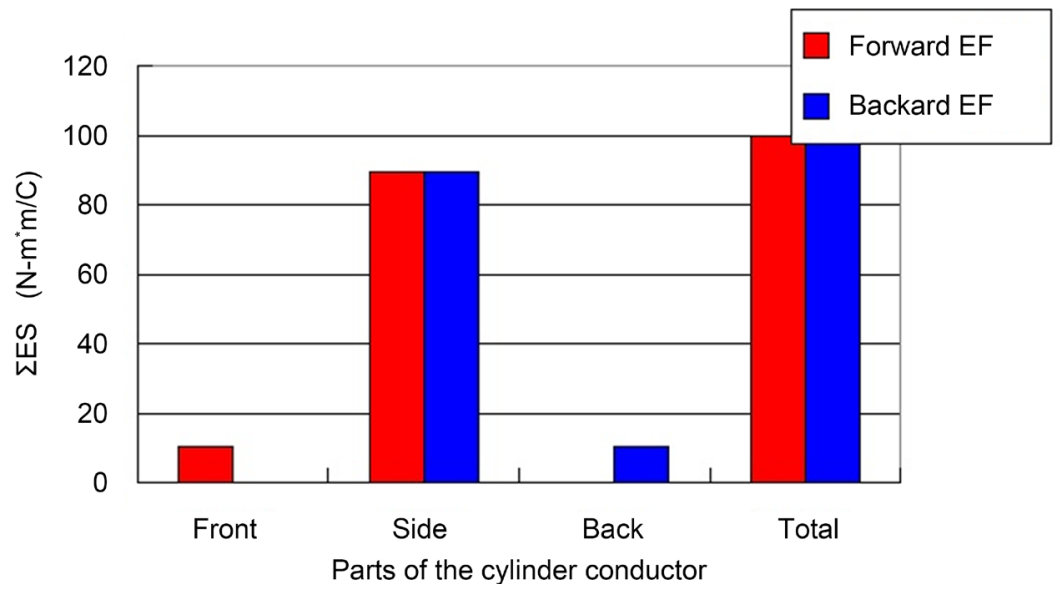

Figure 5. $\Sigma$ ES of each parts of the lonely cylinder shaped charged conductor in the forward and backward electric field.

\subsection{Simulation Result of the Disk-Cylinder Conductor}

Figure 6 shows the simulation result of the disk-cylinder conductor. Total $\Sigma E S$ was 100 too in the both electric field. However $\Sigma E S$ of the disk parts was +110 and $\Sigma E S$ of the cylinder parts was -10 in the forward electric field. This unexpected result will be explained in the next consideration chapter. And $\Sigma E S$ of the disk parts reduced to $16 \%$ and $\Sigma E S$ of the cylinder parts increase to $84 \%$ in the backward electric field. And $\Sigma \mathrm{ES}$ of the disk parts in the forward electric field was mainly consist of the front surface (80\%). And $\Sigma$ ES of the cylinder parts in the backward electric field was mainly consist of the circular side surface $(74 \%)$. Therefore the distribution pattern of $\Sigma$ ES was not right and left symmetric.

Those three simulation results means that if the shape of the conductor is symmetric, the distribution pattern of $\Sigma E S$ becomes symmetric and if the shape of the conductor is not symmetric, the distribution pattern of $\Sigma E S$ does not become symmetric.

\section{Consideration}

\subsection{Charge Distribution Change}

Usually, $\Sigma$ ES is not a familiar physical units for us. Therefore it must be changed to a quantity of electric charge by the following equation [11] for a clear considering.

$$
q_{k}=\sigma_{k} S_{k}=\varepsilon_{0} E_{k} S_{k}
$$

where $\sigma_{\mathrm{k}}$ is surface charge density on the small area of the disk-cylinder conductor, $E_{\mathrm{k}}$ is the intensity of the electric field that through a small area of the Gaussian surface, $S_{\mathrm{k}}$ is area of a small area of the surface of the disk-cylinder conductor, $\varepsilon_{0}$ is Permittivity constant.

Figure 7 shows the distribution of electric charge on the disk-cylinder conductor in the forward and backward electric field. The total quantity of the electric charge is $-0.8854 \mathrm{nC}$. This is the same to the given electric charge. However 


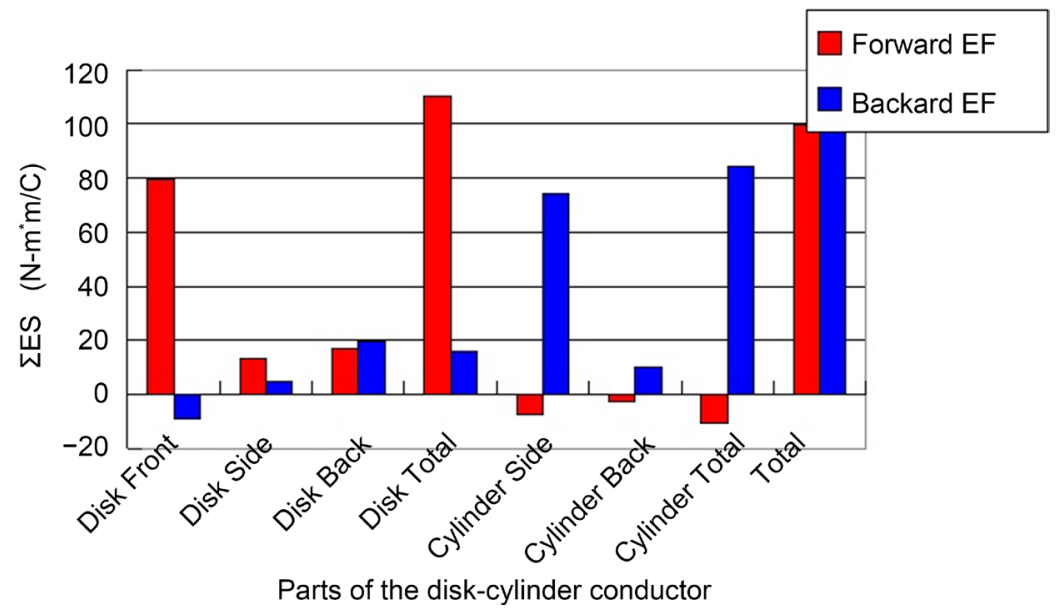

Figure 6. $\Sigma E S$ of each parts of the disk-cylinder shaped charged conductor in the forward and backward electric field.

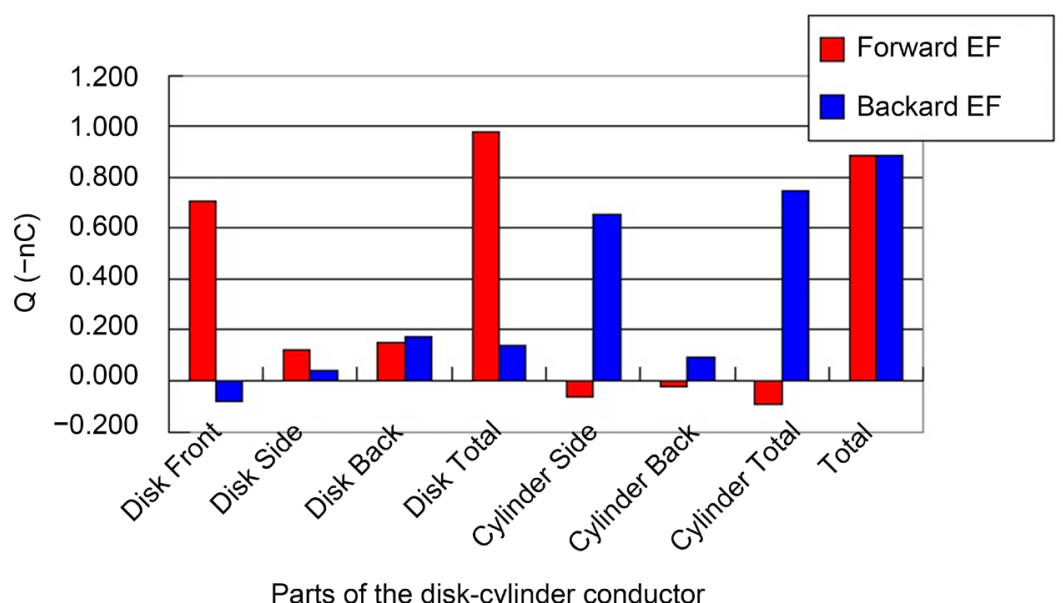

Figure 7. Quantity of charge of each parts of the disk-cylinder shaped charged conductor in the forward and backward electric field.

quantity of charge on the disk in the forward electric field is $-0.977 \mathrm{nC}$. This is larger than quantity of the given electric charge. This difference is clearly explained by an electrostatic induction. Namely $-0.092 \mathrm{nC}$ was added to the disk and $+0.092 \mathrm{nC}$ was added to the cylinder by electrostatic induction in the forward electric field.

It is apparent from this figure that many charge $(-0.71 \mathrm{nC})$ was gathered in the front surface of the disk parts in the forward electric field and many charge $(-0.66 \mathrm{nC})$ was transferred into the circular side surface of the cylinder parts when the direction of the electric field was reversed.

\subsection{Electrostatic Force Distribution Change}

Next, electrostatic forces that acts on the different small area of the disk-cylinder charged conductor were calculated by the following equation [12].

$$
F_{k}=\frac{q_{k} E_{k}}{2}=\frac{\varepsilon_{0} S_{k} E_{k}^{2}}{2}
$$


where $q_{\mathrm{k}}$ is charge on the small area of the disk-cylinder conductor, $E_{\mathrm{k}}$ is the intensity of the electric field that through a small area of the Gaussian surface, $S_{\mathrm{k}}$ is area of a small area of the surface of the disk-cylinder conductor, $\varepsilon_{0}$ is Permittivity constant.

Figure 8 shows the calculation results. It is apparent from this figure that total electrostatic force that acts on the charged disk-cylinder conductor in the forward electric fields is about same to total electrostaitc force in the backward electric field. Namely, the former was $0.57 \mathrm{mN}$ and the latter was $0.52 \mathrm{mN}$. However the electrostatic forces on the $\mathrm{Z}$ direction were different each other. Namely intensity of the electrostatic force in the forward electric field was 0.32 $\mathrm{mN}$ and intensity of the electrostatic force in the backward electric field was 0.17 $\mathrm{mN}$. As mentioned before this big difference between the $\mathrm{Z}$ direction electrostatic forces is called as Asymmetric Electrostatic Force.

The reason of this phenomenon depends on the charge distribution pattern. Many charge $(-0.71 \mathrm{nC})$ gathered into the front surface of the disk parts in the forward electric field and many charge $(-0.66 \mathrm{nC})$ transferred into the circular side surface of the cylinder parts in the backward electric field. As a result, big electrostatic force $(0.38 \mathrm{mN})$ acts on the front surface of the disk parts in the forward electric field and big electrostatic force $(0.31 \mathrm{mN})$ acts on the circular side surface of the cylinder parts in the backward electric field. However the latter force become zero, Because the circular side surface is parallel to the direction of the electric field and the direction of the electric force that acts on this surface is perpendicular to this surface and they cancel each other at an interval of 180 degrees and ultimately become zero.

On the contrary, the front surface of the disk parts is perpendicular to the direction of the electric field and the direction of the electric force that acts on this surface is perpendicular to this surface. As a result this force contributes to the $\mathrm{Z}$ direction electrostatic force $100 \%$.

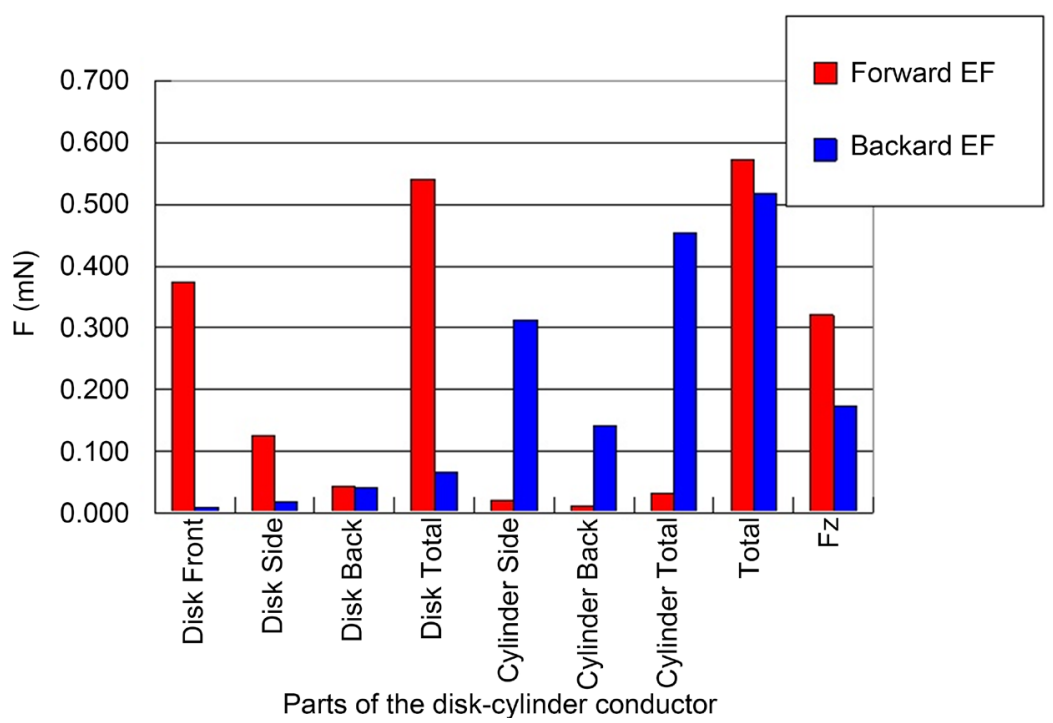

Figure 8. Intensity of the electrostatic force that acts on each parts of the disk-cylinder shaped charged conductor in the forward and backward electric field. 


\section{Conclusion}

Total $\Sigma E S$ on Gaussian surface that encloses the asymmetric shaped charged conductor is the same even if the direction $(\mathrm{Z})$ of the electric field is reversed. And total electrostatic force that acts on this asymmetric shaped charged conductor is about same even if the direction of the electric field is reversed. However the distribution of $\Sigma \mathrm{ES}$ changes when the direction of the electric field is reversed. This result means that the distribution of charge changes. When many charge transferred from the surface that is perpendicular to the direction of the electric field to the other surface that is parallel to the direction of the electric field, the electrostatic force that acts on the former surface contributes $100 \%$ to the $\mathrm{Z}$ direction electrostatic force; on the contrary the electrostatic force that acts on the latter surface does not contributes to the $\mathrm{Z}$ direction electrostatic force. This is the reason of Asymmetric Electrostatic Force.

\section{References}

[1] Halliday, D., Resnick, R. and Walker, J. (2002) Fundamentals of Physics. 6th Edition Japanese Version Chapter "Electric Charge" Question 1, Wiley \& Sons Inc., Hoboken.

[2] Sakai, K. (2008) An Experimental Result which Confirm the Fourth Electrostatic Force. Proceedings of the 2008 ESA Annual Meeting on Electrostatics, Minneapolis, 17-19 June 2008, D3.

[3] Sakai, K. (2009) The Electrostatic Force That Acts on the Charged Asymmetric Conductor in a High Electric Field. 2009 Electrostatics Joint Conference, Boston, 16-18 June 2009, P2.07.

[4] Sakai, K. (2010) Electrostatic Force That Acts on Non-Sphere Shape Charged Conductors. 2010 ESA Annual Meeting, Charlotte, 22-24 June 2010, G4.

[5] Sakai, K. (2011) A Simple Experiment Result That Confirmed Asymmetric Electrostatic Force. 2011 ESA Annual Meeting, Austin, 7-12 August 2011, B4.

[6] Sakai, K. (2010) Asymmetric Electrostatic Forces and a New Electrostatic Generator. Nova Science Publish, New York.

[7] Sakai, K. (2014) Asymmetric Electrostatic Force. Journal of Electromagnetic Analysis and Applications, 6, 253-268. https://doi.org/10.4236/jemaa.2014.610026

[8] Matsubara, Y. (1992) Improved Finite Difference Expression for Laplace's and Poisson's Equations on the Surface of Dielectrics. Journal of the Institute of Electrostatics Japan, 16, 440-442.

[9] Matsubara, Y. (1992) A Guide to the Calculation of Electric Field Part III Application of the Finite Difference Method to Electric Field in an Oil Tank. Journal of the Institute of Electrostatics Japan, 16, 530-538.

[10] Matsubara, Y. (1994) A Method to Calculate the Potential of Ungrounded Conductors. Journal of the Institute of Electrostatics Japan, 181-184.

[11] Halliday, D., Resnick, R. and Walker, J. (2002) Fundamentals of Physics. 6th Edition Japanese Version Chapter “Gauss's Law”, Wiley \& Sons Inc., Hoboken, 39.

[12] Shioda, K. and Chikazumi, S. (1965) Electromagnetism. Shokabo, 25. 
Submit or recommend next manuscript to SCIRP and we will provide best service for you:

Accepting pre-submission inquiries through Email, Facebook, LinkedIn, Twitter, etc. A wide selection of journals (inclusive of 9 subjects, more than 200 journals)

Providing 24-hour high-quality service

User-friendly online submission system

Fair and swift peer-review system

Efficient typesetting and proofreading procedure

Display of the result of downloads and visits, as well as the number of cited articles Maximum dissemination of your research work

Submit your manuscript at: http://papersubmission.scirp.org/

Or contact jemaa@scirp.org 